OPEN $\curvearrowright$ ACCESS

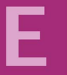
COMMUNICATIONS

ISSN 2056-9890

\section{Crystal structure of 1-nitro-4-(trimethyl- silylethynyl)naphthalene}

\author{
Jun $\mathrm{Du}^{\mathrm{a}}$ and Graeme J. Moxey ${ }^{\mathrm{a}, \mathrm{b} *}$ \\ ${ }^{a}$ School of Chemical and Material Engineering, Jiangnan University, Wuxi, 214122, \\ People's Republic of China, and ${ }^{\mathbf{b}}$ Research School of Chemistry, Australian National \\ University, Canberra, ACT 2601, Australia. *Correspondence e-mail: \\ Graeme.Moxey@anu.edu.au
}

Received 31 March 2015; accepted 10 April 2015

Edited by D.-J. Xu, Zhejiang University (Yuquan Campus), China

In the title compound, $\mathrm{C}_{15} \mathrm{H}_{15} \mathrm{NO}_{2} \mathrm{Si}$, the dihedral angle between the nitro group and the mean plane of the naphthalene system is $22.04(11)^{\circ}$. In the crystal, $\pi-\pi$ interactions generate supramolecular chains propagating along the $a$-axis direction; the centroid-to-centroid distances range from 3.5590 (12) to 3.8535 (12) $\AA$.

Keywords: crystal structure; trialkylsilylacetylene; nitroarene; $\pi-\pi$ interactions.

CCDC reference: 1058939

\section{Related literature}

For the syntheses of arylalkynes by Sonogashira coupling, see: Takahashi et al. (1980). For desilylation of the related 1-nitro4-(trimethylsilylethynyl)benzene and its use in the construction of metal alkynyl complexes with enhanced non-linear optical properties, see: McDonagh et al. (1996a,b, 2003); Garcia et al. (2002). For related structures, see: Squadrito et al. (1990); Khan et al. (2004).

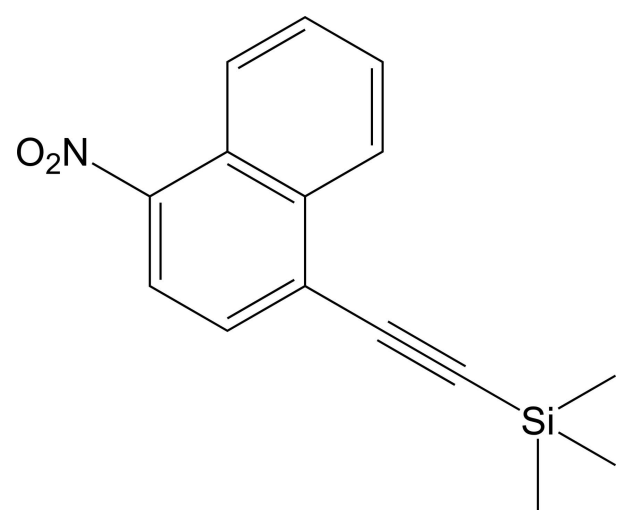

\section{Experimental}

2.1. Crystal data

$\mathrm{C}_{15} \mathrm{H}_{15} \mathrm{NO}_{2} \mathrm{Si}$

$M_{r}=269.37$

Triclinic, $P \overline{1}$

$a=6.9679(9) \AA$

$b=9.2425(12) \AA$

$c=11.799$ (1) $\AA$

$\alpha=100.242(9)^{\circ}$

$\beta=99.698(9)^{\circ}$

$$
\begin{aligned}
& \gamma=107.127(12)^{\circ} \\
& V=694.62(15) \AA^{3} \\
& Z=2 \\
& \text { Mo } K \alpha \text { radiation } \\
& \mu=0.17 \mathrm{~mm}^{-1} \\
& T=150 \mathrm{~K} \\
& 0.23 \times 0.07 \times 0.04 \mathrm{~mm}
\end{aligned}
$$

\subsection{Data collection}

Agilent SuperNova (Dual, Cu at zero, EosS2) diffractometer

Absorption correction: analytical

[CrysAlis PRO (Agilent, 2014),

based on expressions derived by

\author{
Clark \& Reid (1995)] \\ $T_{\text {min }}=0.986, T_{\max }=0.996$ \\ 4695 measured reflections \\ 3112 independent reflections \\ 2621 reflections with $I>2 \sigma(I)$ \\ $R_{\text {int }}=0.021$
}

\subsection{Refinement}

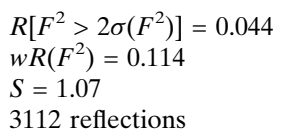

175 parameters $\mathrm{H}$-atom parameters constrained $\Delta \rho_{\max }=0.36{\mathrm{e} \AA^{-3}}^{-3}$ $\Delta \rho_{\min }=-0.23$ e $\AA^{-3}$

Data collection: CrysAlis PRO (Agilent, 2014); cell refinement: CrysAlis PRO; data reduction: CrysAlis PRO; program(s) used to solve structure: SHELXS97 (Sheldrick, 2008); program(s) used to refine structure: SHELXL2013 (Sheldrick, 2015); molecular graphics: OLEX2 (Dolomanov et al., 2009); software used to prepare material for publication: $O L E X 2$.

\section{Acknowledgements}

We gratefully acknowledge support from the Australian Research Council (LE130100057) to purchase Agilent Technologies SuperNova and XCalibur diffractometers. We thank Professors C. Zhang (Jiangnan University), M. P. Cifuentes (Australian National University) and M. G. Humphrey (Australian National University) for assistance.

Supporting information for this paper is available from the $\mathrm{IUCr}$ electronic archives (Reference: XU5846).

\section{References}

Agilent Technologies (2014). CrysAlis PRO. Agilent Technologies, Yarnton, England.

Clark, R. C. \& Reid, J. S. (1995). Acta Cryst. A51, 887-897.

Dolomanov, O. V., Bourhis, L. J., Gildea, R. J., Howard, J. A. K. \& Puschmann, H. (2009). J. Appl. Cryst. 42, 339-341.

Garcia, M. H., Robalo, M. P., Dias, A. R., Duarte, M. T., Wenseleers, W., Aerts, G., Goovaerts, E., Cifuentes, M. P., Hurst, S., Humphrey, M. G., Samoc, M. \& Luther-Davies, B. (2002). Organometallics, 21, 2107-2118.

Khan, M. S., Al-Mandhary, M. R. A., Al-Suti, M. K., Al-Battashi, F. R., AlSaadi, S., Ahrens, B., Bjernemose, J. K., Mahon, M. F., Raithby, P. R., Younus, M., Chawdhury, N., Kohler, A., Marseglia, E. A., Tedesco, E., Feeder, N. \& Teat, S. J. (2004). Dalton Trans. pp. 2377-2385.

McDonagh, A. M., Powell, C. E., Morrall, J. P., Cifuentes, M. P. \& Humphrey, M. G. (2003). Organometallics, 22, 1402-1413. 


\section{data reports}

McDonagh, A. M., Whittall, I. R., Humphrey, M. G., Hockless, D. C. R., Skelton, B. W. \& White, A. H. (1996a). J. Organomet. Chem. 523, 33-40.

McDonagh, A. M., Whittall, I. R., Humphrey, M. G., Skelton, B. W. \& White, A. H. (1996b). J. Organomet. Chem. 519, 229-235.

Sheldrick, G. M. (2008). Acta Cryst. A64, 112-122.
Sheldrick, G. M. (2015). Acta Cryst. C71, 3-8.

Squadrito, G. L., Shane, B. S., Fronczek, F. R., Church, D. F. \& Pryor, W. A. (1990). Chem. Res. Toxicol. 3, 231-235.

Takahashi, S., Kuroyama, Y., Sonogashira, K. \& Hagihara, N. (1980). Synthesis, pp. 627-630. 


\section{supporting information}

Acta Cryst. (2015). E71, o311-o312 [https://doi.org/10.1107/S2056989015007173]

\section{Crystal structure of 1-nitro-4-(trimethylsilylethynyl)naphthalene}

\section{Jun Du and Graeme J. Moxey}

\section{S1. Synthesis and crystallization}

1-Iodo-4-nitronaphthalene $(1.196 \mathrm{~g}, 4.00 \mathrm{mmol})$ was added to triethylamine $(30 \mathrm{~mL})$ and the mixture deoxygenated and charged with nitrogen. $\mathrm{PdCl}_{2}\left(\mathrm{PPh}_{3}\right)_{2}(12 \mathrm{mg}, 0.016 \mathrm{mmol}), \mathrm{CuI}(6 \mathrm{mg}, 0.03 \mathrm{mmol})$ and trimethylsilylacetylene $(0.7 \mathrm{~mL}$, $5.00 \mathrm{mmol}$ ) were added and the reaction heated to $35^{\circ} \mathrm{C}$ overnight. The solution was filtered through filter paper, washing with triethylamine $(10 \mathrm{~mL})$, and the solvent was removed from the filtrate. The residue was then passed through a short pad of silica, eluting with 4:1 petrol: $\mathrm{CH}_{2} \mathrm{Cl}_{2}$. Reduction in volume of the eluate afforded the product as a yellow solid (1.034 g, 96\%). Anal. Calc. for $\mathrm{C}_{15} \mathrm{H}_{15} \mathrm{NO}_{2} \mathrm{Si}$ : C, 66.88; H, 5.61; N, 5.20. Found: C, 66.67; H, 5.68; N, 5.28\%. ${ }^{1} \mathrm{H}$ $\operatorname{NMR}\left(\delta, 400 \mathrm{MHz}, \mathrm{CDCl}_{3}\right): 8.55\left(\mathrm{~d}, J_{\mathrm{HH}}=8.0 \mathrm{~Hz}, 1 \mathrm{H}, \mathrm{H}_{8}\right), 8.47\left(\mathrm{~d}, J_{\mathrm{HH}}=8.0 \mathrm{~Hz}, 1 \mathrm{H}, \mathrm{H}_{5}\right), 8.15\left(\mathrm{~d}, J_{\mathrm{HH}}=8.0 \mathrm{~Hz}, 1 \mathrm{H}\right.$, $\left.\mathrm{H}_{11}\right), 7.79-7.65\left(\mathrm{~m}, 3 \mathrm{H}, \mathrm{H}_{4}, \mathrm{H}_{9}, \mathrm{H}_{10}\right), 0.36$ (s, 9H, Me); ${ }^{13} \mathrm{C}$ NMR $\left(\delta, 101 \mathrm{MHz}, \mathrm{CDCl}_{3}\right): 146.3\left(\mathrm{C}_{6}\right), 134.4\left(\mathrm{C}_{12}\right), 129.8$ $\left(\mathrm{C}_{9}\right), 128.9\left(\mathrm{C}_{4}\right), 128.2\left(\mathrm{C}_{11}\right), 127.7\left(\mathrm{C}_{3}\right), 127.1\left(\mathrm{C}_{10}\right), 125.1\left(\mathrm{C}_{7}\right), 123.5\left(\mathrm{C}_{8}\right), 123.3\left(\mathrm{C}_{5}\right), 105.1\left(\mathrm{C}_{2}\right), 101.4\left(\mathrm{C}_{1}\right), 0.1(\mathrm{~s}$, $\mathrm{Me}$ ); IR (ATR, $\mathrm{cm}^{-1}$ ): 2956, 2156, 1507, 1323. Bright yellow crystals of the title compound were obtained by diffusion of methanol into a dichloromethane solution.

\section{S2. Refinement}

Crystal data, data collection and structure refinement details are summarized below.

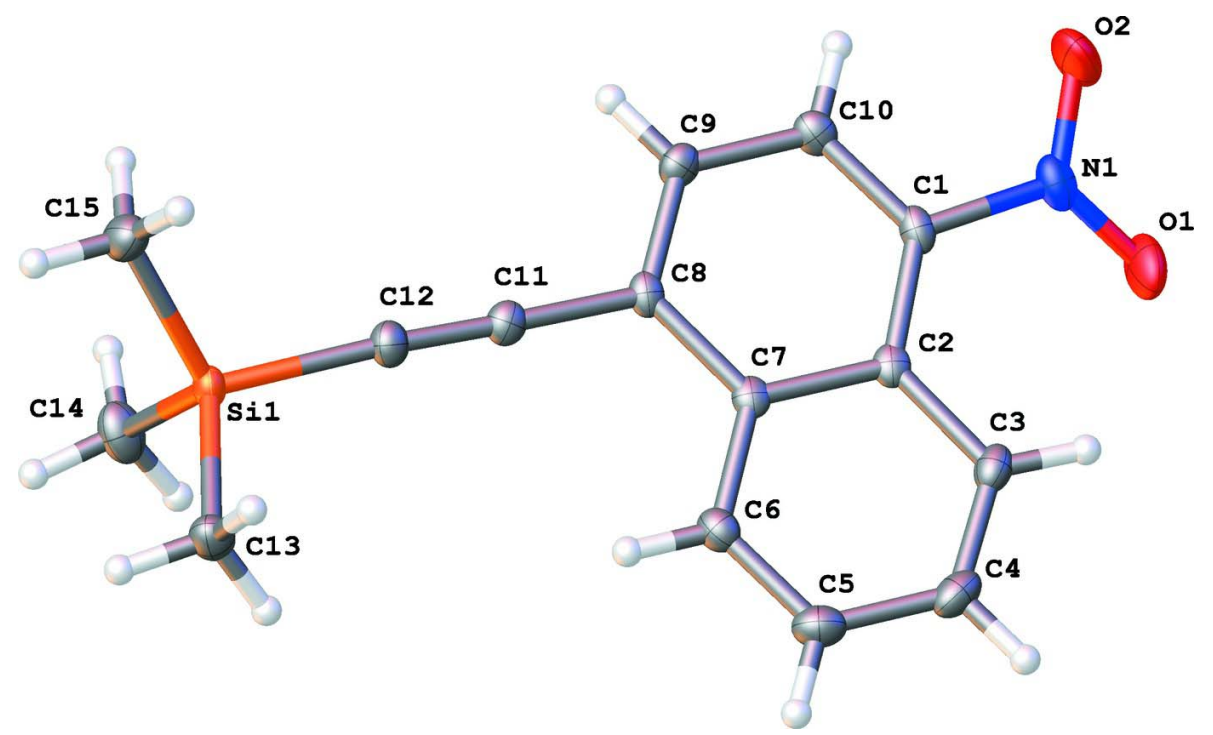

Figure 1

Molecular structure of 1-nitro-4-(trimethylsilylethynyl)naphthalene, with displacement ellipsoids set at the 40\% probability level. 


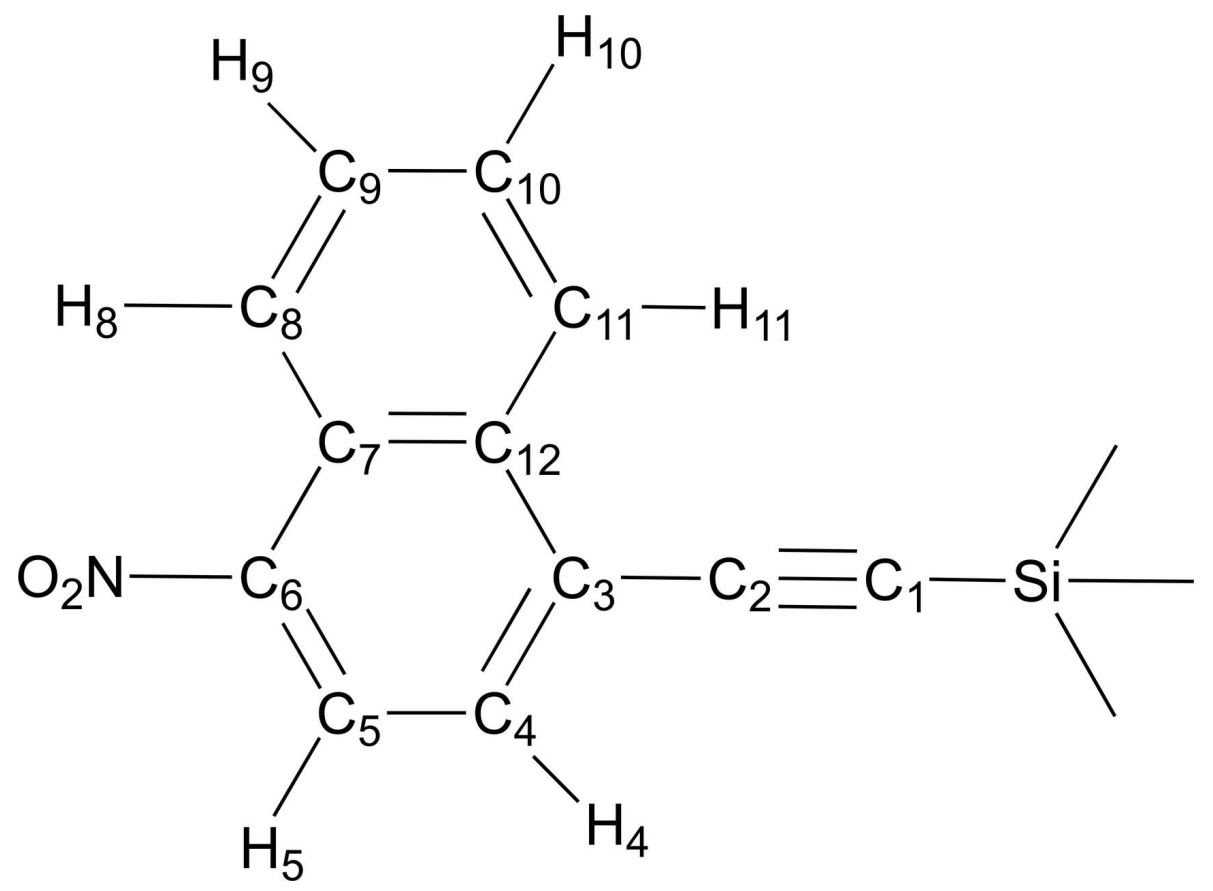

Figure 2

Atom numbering scheme of 1-nitro-4-(trimethylsilylethynyl)naphthalene for ${ }^{1} \mathrm{H}$ and ${ }^{13} \mathrm{C}$ NMR assignments.

1-Nitro-4-(trimethylsilylethynyl)naphthalene

\section{Crystal data}

$\mathrm{C}_{15} \mathrm{H}_{15} \mathrm{NO}_{2} \mathrm{Si}$

$M_{r}=269.37$

Triclinic, $P \overline{1}$

$a=6.9679(9) \AA$

$b=9.2425(12) \AA$

$c=11.799(1) \AA$

$\alpha=100.242(9)^{\circ}$

$\beta=99.698(9)^{\circ}$

$\gamma=107.127(12)^{\circ}$

$V=694.62(15) \AA^{3}$

\section{Data collection}

Agilent SuperNova (Dual, Cu at zero, EosS2) diffractometer

Radiation source: SuperNova (Mo) X-ray Source

Mirror monochromator

Detector resolution: 8.1297 pixels $\mathrm{mm}^{-1}$

$\omega$ scans

Absorption correction: analytical

[CrysAlis PRO (Agilent, 2014), based on

expressions derived by Clark \& Reid (1995)]
$Z=2$

$F(000)=284$

$D_{\mathrm{x}}=1.288 \mathrm{Mg} \mathrm{m}^{-3}$

Mo $K \alpha$ radiation, $\lambda=0.71073 \AA$

Cell parameters from 1967 reflections

$\theta=2.6-28.3^{\circ}$

$\mu=0.17 \mathrm{~mm}^{-1}$

$T=150 \mathrm{~K}$

Needle, yellow

$0.23 \times 0.07 \times 0.04 \mathrm{~mm}$

$T_{\min }=0.986, T_{\max }=0.996$

4695 measured reflections

3112 independent reflections

2621 reflections with $I>2 \sigma(I)$

$R_{\text {int }}=0.021$

$\theta_{\text {max }}=29.2^{\circ}, \theta_{\min }=1.8^{\circ}$

$h=-6 \rightarrow 9$

$k=-11 \rightarrow 12$

$l=-15 \rightarrow 15$ 


\section{Refinement}

Refinement on $F^{2}$

Least-squares matrix: full

$R\left[F^{2}>2 \sigma\left(F^{2}\right)\right]=0.044$

$w R\left(F^{2}\right)=0.114$

$S=1.07$

3112 reflections

175 parameters

0 restraints
Primary atom site location: structure-invariant direct methods

Hydrogen site location: inferred from neighbouring sites

$\mathrm{H}$-atom parameters constrained

$w=1 /\left[\sigma^{2}\left(F_{\mathrm{o}}^{2}\right)+(0.0415 P)^{2}+0.3469 P\right]$ where $P=\left(F_{\mathrm{o}}{ }^{2}+2 F_{\mathrm{c}}{ }^{2}\right) / 3$

$(\Delta / \sigma)_{\max }<0.001$

$\Delta \rho_{\max }=0.36 \mathrm{e} \AA^{-3}$

$\Delta \rho_{\min }=-0.23$ e $\AA^{-3}$

Special details

Experimental. Absorption correction: CrysAlis Pro (Agilent Technologies, 2014) Analytical numeric absorption correction using a multifaceted crystal model based on expressions derived by R.C. Clark \& J.S. Reid. (Clark \& Reid, 1995). Empirical absorption correction using spherical harmonics, implemented in SCALE3 ABSPACK scaling algorithm.

Geometry. All e.s.d.'s (except the e.s.d. in the dihedral angle between two l.s. planes) are estimated using the full covariance matrix. The cell e.s.d.'s are taken into account individually in the estimation of e.s.d.'s in distances, angles and torsion angles; correlations between e.s.d.'s in cell parameters are only used when they are defined by crystal symmetry. An approximate (isotropic) treatment of cell e.s.d.'s is used for estimating e.s.d.'s involving 1.s. planes.

Fractional atomic coordinates and isotropic or equivalent isotropic displacement parameters $\left(\hat{A}^{2}\right)$

\begin{tabular}{|c|c|c|c|c|}
\hline & $x$ & $y$ & $z$ & $U_{\text {iso }} * / U_{\text {eq }}$ \\
\hline $\mathrm{C} 1$ & 0.2485 & $0.6318(2)$ & $0.44838(15)$ & $0.0204(4)$ \\
\hline $\mathrm{C} 2$ & $0.1929(2)$ & $0.4663(2)$ & $0.41137(15)$ & $0.0188(4)$ \\
\hline $\mathrm{C} 3$ & $0.1112(3)$ & $0.3754(2)$ & $0.29263(16)$ & $0.0247(4)$ \\
\hline $\mathrm{H} 3$ & 0.0875 & 0.4239 & 0.2316 & $0.030^{*}$ \\
\hline $\mathrm{C} 4$ & 0.0675 & $0.2178(2)$ & $0.26757(17)$ & $0.0293(4)$ \\
\hline $\mathrm{H} 4$ & 0.0147 & 0.1606 & 0.1893 & $0.035^{*}$ \\
\hline $\mathrm{C} 5$ & $0.1001(3)$ & $0.1400(2)$ & $0.35659(18)$ & $0.0293(4)$ \\
\hline H5 & 0.0661 & 0.0321 & 0.3376 & $0.035^{*}$ \\
\hline C6 & 0.1819 & 0.2230 & $0.47120(17)$ & $0.0234(4)$ \\
\hline H6 & 0.2048 & 0.1711 & 0.5301 & $0.028 *$ \\
\hline $\mathrm{C} 7$ & 0.2324 & $0.3874(2)$ & $0.50191(15)$ & $0.0182(4)$ \\
\hline $\mathrm{C} 8$ & 0.3257 & 0.4749 & $0.62159(15)$ & $0.0187(4)$ \\
\hline $\mathrm{C} 9$ & 0.3745 & $0.6352(2)$ & $0.65052(15)$ & $0.0220(4)$ \\
\hline H9 & 0.4329 & 0.6912 & 0.7288 & $0.026^{*}$ \\
\hline $\mathrm{C} 10$ & 0.3369 & $0.7131(2)$ & $0.56329(16)$ & $0.0225(4)$ \\
\hline H10 & 0.3720 & 0.8211 & 0.5832 & $0.027^{*}$ \\
\hline C11 & $0.3756(3)$ & $0.3974(2)$ & $0.71220(15)$ & $0.0215(4)$ \\
\hline $\mathrm{C} 12$ & $0.4216(3)$ & $0.3354(2)$ & $0.78877(16)$ & $0.0235(4)$ \\
\hline $\mathrm{C} 13$ & $0.4757(3)$ & $0.0381(2)$ & $0.83030(17)$ & $0.0300(4)$ \\
\hline $\mathrm{H} 13 \mathrm{~A}$ & 0.3530 & -0.0142 & 0.7682 & $0.045^{*}$ \\
\hline H13B & 0.4823 & -0.0237 & 0.8873 & $0.045^{*}$ \\
\hline $\mathrm{H} 13 \mathrm{C}$ & 0.5949 & 0.0523 & 0.7973 & $0.045^{*}$ \\
\hline $\mathrm{C} 14$ & 0.2518 & 0.2089 & $0.97894(19)$ & $0.0361(5)$ \\
\hline $\mathrm{H} 14 \mathrm{~A}$ & 0.1234 & 0.1699 & 0.9203 & $0.054^{*}$ \\
\hline H14B & 0.2647 & 0.3083 & 1.0269 & $0.054^{*}$ \\
\hline
\end{tabular}




$\begin{array}{lllll}\text { H14C } & 0.2547 & 0.1369 & 1.0281 & 0.054^{*} \\ \text { C15 } & 0.7217(3) & 0.3443(2) & 1.00937(18) & 0.0327(5) \\ \text { H15A } & 0.7507 & 0.2860 & 1.0658 & 0.049^{*} \\ \text { H15B } & 0.7161 & 0.4421 & 1.0501 & 0.049^{*} \\ \text { H15C } & 0.8287 & 0.3633 & 0.9667 & 0.049^{*} \\ \text { N1 } & 0.2165(3) & 0.7285(2) & 0.36481(15) & 0.0275(4) \\ \text { O1 } & 0.0903(2) & 0.6698(2) & 0.27072(14) & 0.0437(4) \\ \text { O2 } & 0.3213(3) & 0.86743(19) & 0.39546(15) & 0.0532(5) \\ \text { Si1 } & 0.47002(8) & 0.23164(6) & 0.90416(4) & 0.02062(14)\end{array}$

Atomic displacement parameters $\left(\AA^{2}\right)$

\begin{tabular}{lllllll}
\hline & $U^{11}$ & $U^{22}$ & $U^{33}$ & $U^{12}$ & $U^{13}$ & $U^{23}$ \\
\hline C1 & $0.0176(8)$ & $0.0279(10)$ & $0.0240(9)$ & $0.0126(7)$ & $0.0093(7)$ & $0.0138(7)$ \\
C2 & $0.0125(7)$ & $0.0262(9)$ & $0.0212(8)$ & $0.0085(7)$ & $0.0063(7)$ & $0.0086(7)$ \\
C3 & $0.0193(9)$ & $0.0349(11)$ & $0.0191(9)$ & $0.0081(8)$ & $0.0036(7)$ & $0.0076(8)$ \\
C4 & $0.0228(9)$ & $0.0372(12)$ & $0.0213(9)$ & $0.0066(8)$ & $0.0030(8)$ & $-0.0012(8)$ \\
C5 & $0.0256(10)$ & $0.0240(10)$ & $0.0350(11)$ & $0.0071(8)$ & $0.0065(8)$ & $0.0016(8)$ \\
C6 & $0.0211(9)$ & $0.0239(10)$ & $0.0280(9)$ & $0.0093(7)$ & $0.0067(8)$ & $0.0093(8)$ \\
C7 & $0.0123(7)$ & $0.0238(9)$ & $0.0212(8)$ & $0.0077(7)$ & $0.0064(7)$ & $0.0074(7)$ \\
C8 & $0.0160(8)$ & $0.0264(9)$ & $0.0199(8)$ & $0.0114(7)$ & $0.0078(7)$ & $0.0100(7)$ \\
C9 & $0.0217(9)$ & $0.0262(10)$ & $0.0193(8)$ & $0.0103(7)$ & $0.0065(7)$ & $0.0033(7)$ \\
C10 & $0.0233(9)$ & $0.0229(9)$ & $0.0267(9)$ & $0.0124(8)$ & $0.0105(8)$ & $0.0073(7)$ \\
C11 & $0.0196(8)$ & $0.0267(10)$ & $0.0208(9)$ & $0.0105(7)$ & $0.0070(7)$ & $0.0054(7)$ \\
C12 & $0.0248(9)$ & $0.0274(10)$ & $0.0217(9)$ & $0.0110(8)$ & $0.0078(7)$ & $0.0083(7)$ \\
C13 & $0.0396(11)$ & $0.0241(10)$ & $0.0272(10)$ & $0.0113(9)$ & $0.0099(9)$ & $0.0058(8)$ \\
C14 & $0.0410(12)$ & $0.0438(13)$ & $0.0373(11)$ & $0.0215(10)$ & $0.0217(10)$ & $0.0197(10)$ \\
C15 & $0.0364(11)$ & $0.0296(11)$ & $0.0282(10)$ & $0.0100(9)$ & $-0.0004(9)$ & $0.0066(8)$ \\
N1 & $0.0294(9)$ & $0.0352(10)$ & $0.0313(9)$ & $0.0193(8)$ & $0.0158(7)$ & $0.0186(8)$ \\
O1 & $0.0390(9)$ & $0.0558(11)$ & $0.0400(9)$ & $0.0167(8)$ & $-0.0007(7)$ & $0.0290(8)$ \\
O2 & $0.0889(14)$ & $0.0287(9)$ & $0.0447(10)$ & $0.0188(9)$ & $0.0129(9)$ & $0.0198(8)$ \\
Si1 & $0.0252(3)$ & $0.0222(3)$ & $0.0169(2)$ & $0.0097(2)$ & $0.00518(19)$ & $0.00766(19)$ \\
& & & & & &
\end{tabular}

Geometric parameters $\left(\AA,{ }^{\circ}\right)$

\begin{tabular}{llll}
\hline $\mathrm{C} 1-\mathrm{C} 2$ & $1.427(3)$ & $\mathrm{C} 10-\mathrm{H} 10$ & 0.9300 \\
$\mathrm{C} 1-\mathrm{C} 10$ & $1.366(3)$ & $\mathrm{C} 11-\mathrm{C} 12$ & $1.201(2)$ \\
$\mathrm{C} 1-\mathrm{N} 1$ & $1.476(2)$ & $\mathrm{C} 12-\mathrm{Si} 1$ & $1.8403(19)$ \\
$\mathrm{C} 2-\mathrm{C} 3$ & $1.422(3)$ & $\mathrm{C} 13-\mathrm{H} 13 \mathrm{~A}$ & 0.9600 \\
$\mathrm{C} 2-\mathrm{C} 7$ & $1.430(2)$ & $\mathrm{C} 13-\mathrm{H} 13 \mathrm{~B}$ & 0.9600 \\
$\mathrm{C} 3-\mathrm{H} 3$ & 0.9300 & $\mathrm{C} 13-\mathrm{H} 13 \mathrm{C}$ & 0.9600 \\
$\mathrm{C} 3-\mathrm{C} 4$ & $1.363(3)$ & $\mathrm{C} 13-\mathrm{Si} 1$ & $1.860(2)$ \\
$\mathrm{C} 4-\mathrm{H} 4$ & 0.9300 & $\mathrm{C} 14-\mathrm{H} 14 \mathrm{~A}$ & 0.9600 \\
$\mathrm{C} 4-\mathrm{C} 5$ & $1.399(3)$ & $\mathrm{C} 14-\mathrm{H} 14 \mathrm{~B}$ & 0.9600 \\
$\mathrm{C} 5-\mathrm{H} 5$ & 0.9300 & $\mathrm{C} 14-\mathrm{H} 14 \mathrm{C}$ & 0.9600 \\
$\mathrm{C} 5-\mathrm{C} 6$ & $1.362(3)$ & $\mathrm{C} 14-\mathrm{Si} 1$ & $1.862(2)$ \\
$\mathrm{C} 6-\mathrm{H} 6$ & 0.9300 & $\mathrm{C} 15-\mathrm{H} 15 \mathrm{~A}$ & 0.9600 \\
$\mathrm{C} 6-\mathrm{C} 7$ & $1.418(3)$ & $\mathrm{C} 15-\mathrm{H} 15 \mathrm{~B}$ & 0.9600
\end{tabular}




\begin{tabular}{|c|c|c|c|}
\hline $\mathrm{C} 7-\mathrm{C} 8$ & $1.430(2)$ & $\mathrm{C} 15-\mathrm{H} 15 \mathrm{C}$ & 0.9600 \\
\hline $\mathrm{C} 8-\mathrm{C} 9$ & $1.382(3)$ & $\mathrm{C} 15-\mathrm{Si} 1$ & $1.853(2)$ \\
\hline $\mathrm{C} 8-\mathrm{C} 11$ & $1.439(2)$ & $\mathrm{N} 1-\mathrm{O} 1$ & $1.215(2)$ \\
\hline $\mathrm{C} 9-\mathrm{H} 9$ & 0.9300 & $\mathrm{~N} 1-\mathrm{O} 2$ & $1.228(2)$ \\
\hline $\mathrm{C} 9-\mathrm{C} 10$ & $1.390(2)$ & & \\
\hline $\mathrm{C} 2-\mathrm{C} 1-\mathrm{N} 1$ & $122.38(16)$ & $\mathrm{C} 12-\mathrm{C} 11-\mathrm{C} 8$ & $178.5(2)$ \\
\hline $\mathrm{C} 10-\mathrm{C} 1-\mathrm{C} 2$ & $122.82(16)$ & $\mathrm{C} 11-\mathrm{C} 12-\mathrm{Si} 1$ & $175.42(17)$ \\
\hline $\mathrm{C} 10-\mathrm{C} 1-\mathrm{N} 1$ & $114.80(16)$ & $\mathrm{H} 13 \mathrm{~A}-\mathrm{C} 13-\mathrm{H} 13 \mathrm{~B}$ & 109.5 \\
\hline $\mathrm{C} 1-\mathrm{C} 2-\mathrm{C} 7$ & $116.38(15)$ & $\mathrm{H} 13 \mathrm{~A}-\mathrm{C} 13-\mathrm{H} 13 \mathrm{C}$ & 109.5 \\
\hline $\mathrm{C} 3-\mathrm{C} 2-\mathrm{C} 1$ & $125.72(16)$ & $\mathrm{H} 13 \mathrm{~B}-\mathrm{C} 13-\mathrm{H} 13 \mathrm{C}$ & 109.5 \\
\hline $\mathrm{C} 3-\mathrm{C} 2-\mathrm{C} 7$ & $117.84(17)$ & Sil $-\mathrm{C} 13-\mathrm{H} 13 \mathrm{~A}$ & 109.5 \\
\hline $\mathrm{C} 2-\mathrm{C} 3-\mathrm{H} 3$ & 119.7 & Sil-C13-H13B & 109.5 \\
\hline $\mathrm{C} 4-\mathrm{C} 3-\mathrm{C} 2$ & $120.51(17)$ & Si1-C13-H13C & 109.5 \\
\hline $\mathrm{C} 4-\mathrm{C} 3-\mathrm{H} 3$ & 119.7 & $\mathrm{H} 14 \mathrm{~A}-\mathrm{C} 14-\mathrm{H} 14 \mathrm{~B}$ & 109.5 \\
\hline $\mathrm{C} 3-\mathrm{C} 4-\mathrm{H} 4$ & 119.2 & $\mathrm{H} 14 \mathrm{~A}-\mathrm{C} 14-\mathrm{H} 14 \mathrm{C}$ & 109.5 \\
\hline $\mathrm{C} 3-\mathrm{C} 4-\mathrm{C} 5$ & $121.65(18)$ & $\mathrm{H} 14 \mathrm{~B}-\mathrm{C} 14-\mathrm{H} 14 \mathrm{C}$ & 109.5 \\
\hline $\mathrm{C} 5-\mathrm{C} 4-\mathrm{H} 4$ & 119.2 & Si1-C14-H14A & 109.5 \\
\hline $\mathrm{C} 4-\mathrm{C} 5-\mathrm{H} 5$ & 120.1 & $\mathrm{Si} 1-\mathrm{C} 14-\mathrm{H} 14 \mathrm{~B}$ & 109.5 \\
\hline $\mathrm{C} 6-\mathrm{C} 5-\mathrm{C} 4$ & $119.74(18)$ & Sil-C14-H14C & 109.5 \\
\hline $\mathrm{C} 6-\mathrm{C} 5-\mathrm{H} 5$ & 120.1 & $\mathrm{H} 15 \mathrm{~A}-\mathrm{C} 15-\mathrm{H} 15 \mathrm{~B}$ & 109.5 \\
\hline $\mathrm{C} 5-\mathrm{C} 6-\mathrm{H} 6$ & 119.6 & $\mathrm{H} 15 \mathrm{~A}-\mathrm{C} 15-\mathrm{H} 15 \mathrm{C}$ & 109.5 \\
\hline $\mathrm{C} 5-\mathrm{C} 6-\mathrm{C} 7$ & $120.89(17)$ & $\mathrm{H} 15 \mathrm{~B}-\mathrm{C} 15-\mathrm{H} 15 \mathrm{C}$ & 109.5 \\
\hline $\mathrm{C} 7-\mathrm{C} 6-\mathrm{H} 6$ & 119.6 & Sil $-\mathrm{C} 15-\mathrm{H} 15 \mathrm{~A}$ & 109.5 \\
\hline $\mathrm{C} 2-\mathrm{C} 7-\mathrm{C} 8$ & $119.87(16)$ & Si1-C15-H15B & 109.5 \\
\hline $\mathrm{C} 6-\mathrm{C} 7-\mathrm{C} 2$ & $119.33(16)$ & Si1-C15-H15C & 109.5 \\
\hline $\mathrm{C} 6-\mathrm{C} 7-\mathrm{C} 8$ & $120.79(16)$ & $\mathrm{O} 1-\mathrm{N} 1-\mathrm{C} 1$ & $119.96(17)$ \\
\hline $\mathrm{C} 7-\mathrm{C} 8-\mathrm{C} 11$ & $120.25(16)$ & $\mathrm{O} 1-\mathrm{N} 1-\mathrm{O} 2$ & $123.02(17)$ \\
\hline $\mathrm{C} 9-\mathrm{C} 8-\mathrm{C} 7$ & $120.30(16)$ & $\mathrm{O} 2-\mathrm{N} 1-\mathrm{C} 1$ & $117.02(17)$ \\
\hline $\mathrm{C} 9-\mathrm{C} 8-\mathrm{C} 11$ & $119.42(16)$ & $\mathrm{C} 12-\mathrm{Si} 1-\mathrm{C} 13$ & $107.97(9)$ \\
\hline $\mathrm{C} 8-\mathrm{C} 9-\mathrm{H} 9$ & 119.8 & $\mathrm{C} 12-\mathrm{Si} 1-\mathrm{C} 14$ & $106.63(9)$ \\
\hline $\mathrm{C} 8-\mathrm{C} 9-\mathrm{C} 10$ & $120.33(16)$ & $\mathrm{C} 12-\mathrm{Si} 1-\mathrm{C} 15$ & $109.92(9)$ \\
\hline $\mathrm{C} 10-\mathrm{C} 9-\mathrm{H} 9$ & 119.8 & $\mathrm{C} 13-\mathrm{Si} 1-\mathrm{C} 14$ & $110.88(10)$ \\
\hline $\mathrm{C} 1-\mathrm{C} 10-\mathrm{C} 9$ & $120.29(17)$ & $\mathrm{C} 15-\mathrm{Si} 1-\mathrm{C} 13$ & $109.63(10)$ \\
\hline $\mathrm{C} 1-\mathrm{C} 10-\mathrm{H} 10$ & 119.9 & $\mathrm{C} 15-\mathrm{Si} 1-\mathrm{C} 14$ & $111.70(10)$ \\
\hline $\mathrm{C} 9-\mathrm{C} 10-\mathrm{H} 10$ & 119.9 & & \\
\hline $\mathrm{C} 1-\mathrm{C} 2-\mathrm{C} 3-\mathrm{C} 4$ & $178.69(16)$ & $\mathrm{C} 5-\mathrm{C} 6-\mathrm{C} 7-\mathrm{C} 8$ & $-177.53(16)$ \\
\hline $\mathrm{C} 1-\mathrm{C} 2-\mathrm{C} 7-\mathrm{C} 6$ & $-179.69(14)$ & $\mathrm{C} 6-\mathrm{C} 7-\mathrm{C} 8-\mathrm{C} 9$ & $179.94(15)$ \\
\hline $\mathrm{C} 1-\mathrm{C} 2-\mathrm{C} 7-\mathrm{C} 8$ & $-0.9(2)$ & $\mathrm{C} 6-\mathrm{C} 7-\mathrm{C} 8-\mathrm{C} 11$ & $1.9(2)$ \\
\hline $\mathrm{C} 2-\mathrm{C} 1-\mathrm{C} 10-\mathrm{C} 9$ & $-0.7(3)$ & $\mathrm{C} 7-\mathrm{C} 2-\mathrm{C} 3-\mathrm{C} 4$ & $1.7(2)$ \\
\hline $\mathrm{C} 2-\mathrm{C} 1-\mathrm{N} 1-\mathrm{O} 1$ & $21.6(2)$ & $\mathrm{C} 7-\mathrm{C} 8-\mathrm{C} 9-\mathrm{C} 10$ & $-1.2(2)$ \\
\hline $\mathrm{C} 2-\mathrm{C} 1-\mathrm{N} 1-\mathrm{O} 2$ & $-158.72(17)$ & $\mathrm{C} 8-\mathrm{C} 9-\mathrm{C} 10-\mathrm{C} 1$ & $0.9(3)$ \\
\hline $\mathrm{C} 2-\mathrm{C} 3-\mathrm{C} 4-\mathrm{C} 5$ & $0.2(3)$ & $\mathrm{C} 10-\mathrm{C} 1-\mathrm{C} 2-\mathrm{C} 3$ & $-176.32(16)$ \\
\hline $\mathrm{C} 2-\mathrm{C} 7-\mathrm{C} 8-\mathrm{C} 9$ & $1.2(2)$ & $\mathrm{C} 10-\mathrm{C} 1-\mathrm{C} 2-\mathrm{C} 7$ & $0.7(2)$ \\
\hline $\mathrm{C} 2-\mathrm{C} 7-\mathrm{C} 8-\mathrm{C} 11$ & $-176.86(14)$ & $\mathrm{C} 10-\mathrm{C} 1-\mathrm{N} 1-\mathrm{O} 1$ & $-158.99(17)$ \\
\hline $\mathrm{C} 3-\mathrm{C} 2-\mathrm{C} 7-\mathrm{C} 6$ & $-2.4(2)$ & $\mathrm{C} 10-\mathrm{C} 1-\mathrm{N} 1-\mathrm{O} 2$ & $20.7(2)$ \\
\hline $\mathrm{C} 3-\mathrm{C} 2-\mathrm{C} 7-\mathrm{C} 8$ & $176.36(15)$ & $\mathrm{C} 11-\mathrm{C} 8-\mathrm{C} 9-\mathrm{C} 10$ & $176.88(15)$ \\
\hline
\end{tabular}


$\mathrm{C} 3-\mathrm{C} 4-\mathrm{C} 5-\mathrm{C} 6$
$\mathrm{C} 4-\mathrm{C} 5-\mathrm{C} 6-\mathrm{C} 7$
$\mathrm{C} 5-\mathrm{C} 6-\mathrm{C} 7-\mathrm{C} 2$

$-1.5(3)$

0.7 (3)

$1.2(2)$
$\mathrm{N} 1-\mathrm{C} 1-\mathrm{C} 2-\mathrm{C} 3$

$\mathrm{N} 1-\mathrm{C} 1-\mathrm{C} 2-\mathrm{C} 7$

$\mathrm{N} 1-\mathrm{C} 1-\mathrm{C} 10-\mathrm{C} 9$
$3.1(3)$

$-179.89(14)$

$179.83(14)$ 